10. - A necessary condition that a cellular upper semicontinuous decomposiion of $E^{n}$ yield $E^{n}$, Trans. Amer. Math. Soc. 122 (1966), 427-435.

11. - Decompositions of $\mathrm{S}^{3}$ and pseudo-isotopies, Notices Amer. Math. Soc. 15 (1968), 103.

12. W. Voxman, Decompositions of 3-manifolds and pseudo-isotopies, Notices Amer. Math. Soc. 15 (1968), 547.

13. - On the shrinkability of decompositions of 3-manifolds, Notices Amer. Math. Soc. 15 (1968), 649.

University of Iowa, Iowa City, Iowa 52240

\title{
ON ONE PARAMETER FAMILIES OF REAL SOLUTIONS OF NONLINEAR OPERATOR EQUATIONS
}

\author{
BY MELVYN S. BERGER ${ }^{1}$
}

Communicated by Avner Friedman, November 6, 1968

Let $H$ be a separable Hilbert space over the real numbers. Denote by $L, M, N$ bounded mappings of $H$ into itself. In an earlier note [1] the author studied the solutions $u \in H$ of operator equations of the form

$$
u+M u=\lambda\{L u+N u\}
$$

under the assumption that such solutions be contained in a sufficiently small sphere with center at $u=0$. Here we assume that $L, M$, and $N$ are odd, completely continuous, locally Lipschitzian gradient mappings of $H$ into itself, and $\lambda$ is a real parameter. We wish to announce some results concerning the global structure of solutions of (1) and some applications of these results to problems arising in the calculus of variations. Proofs of these results will appear elsewhere.

1. Statements of results. Suppose $\mathfrak{M}$ and $\mathfrak{N}$ are even functionals such that grad $\mathfrak{M}=M$ and $\operatorname{grad} \mathfrak{N}=N$, then we set $F(u)$ $=(1 / 2)\|u\|^{2}+\mathfrak{M}(u) \quad$ and $\quad G(u)=(1 / 2)(L u, u)+\mathfrak{N}(u), \quad$ and $\quad \partial A_{R}$ $=\{u \mid F(u)=R, R$ a fixed real number $\}$. Associated with (1) we consider its linearization at $u=0$

$$
u=\lambda L u \text {. }
$$

We assume $(L u, u)>0$ for $u \neq 0$ so that the eigenvalues of (2) $\left\{\lambda_{i}\right\}$ form an increasing sequence of real numbers $0<\lambda_{1} \leqq \lambda_{2} \leqq \lambda_{3} \cdots$.

\footnotetext{
${ }^{2}$ Research partially supported by NSF GP-7041X.
} 
Concerning the operators $M$ and $N$ we assume for each $R, 0<R \leqq R_{0}$,

(i) $\partial A_{R}$ is a manifold in $H$, i.e., $\operatorname{grad} F(u) \neq 0$ for $u \in \partial A_{R}$;

(ii) $\partial A_{R}$ is starlike and homeomorphic to the sphere $\|u\|=1$ along rays through the origin;

(iii) $(L u+N u, u)>0$ for $u \neq 0$. Also $\|N u-N v\|=o(\|u-v\|)$.

Then the following result enables us to establish global one parameter families of solutions for Equation (1).

TheOREM 1 (Continuation THEOREM). Under the above assumptions suppose $\lambda_{n}$ is an eigenvalue of multiplicity $p$ of Equation (2), then Equation (1) has at least $p$ distinct one parameter families of solutions $\left(u_{n, i}(R), \quad \lambda_{n, i}(R)\right)(i=1, \cdots, p)$ with $u_{n, i}(R) \in \partial A_{R}$, such that $\left(u_{n, i}(R), \quad \lambda_{n, i}(R)\right) \rightarrow\left(0, \lambda_{n}\right)$ as $R \rightarrow 0$. Furthermore the families $\left(u_{n, i}(R), \lambda_{n, i}(R)\right)$ can be continued as functions of $R$ to the interval $\left(0, R_{0}\right)$ by means of the characterization

$$
G\left(u_{n, i}(R)\right)=\sup _{[V]_{n, i} V} \min G(u)
$$

where $[V]_{n, i}$ is an appropriate isotopy class of sets on $\partial A_{R} / Z_{2}$.

We next attempt to answer the question: "Under what circumstances are the families $\left(u_{n, i}(R), \lambda_{n, i}(R)\right)$ continuous with respect to $R$ ?" To this end we recall that an operator $Q(u)$ is real analytic if $Q(u+t v)$ is a real analytic function of $t$ for all $u, v \in H$ in a small neighborhood of the origin (see [3, Chapter 3$]$ ).

THEOREM 2 (CONTINUITY THEOREM). If in addition to the hypotheses of Theorem 1, we suppose $M=0$ and $N$ is real analytic then the one parameter families mentioned in Theorem $1 \lambda_{n, i}(R)$ are continuous functions of $R$ for $R$ sufficiently small.

REMARKS ON PROOF. Theorem 1 is obtained by means of the Ljusternik-Schnirelmann theory of critical points on hypersurfaces in $H$. Theorem 2 is obtained by combining the theory of real analytic varieties of Bruhat and Whitney, with the bifurcation theory of E. Schmidt.

2. Application to periodic solutions of Hamiltonian systems. Let $A$ be an $n \times n$ positive-definite selfadjoint matrix with eigenvalues $\left\{\beta_{i}^{2}\right\}$, $0<\beta_{1}^{2} \leqq \beta_{2}^{2} \leqq \cdots \leqq \beta_{n}^{2}, x(t)$ be an $n$-vector function of the real variable $t$, and $f(x)=\operatorname{grad} F(x)$ be the gradient of the $C^{2}$ real-valued analytic function $F(x)$. Then we consider the periodic solutions of the system

$$
\ddot{x}+A x+f(x)=0 \text {. }
$$


The following result extends a well-known result of Liyapunov to the case of "resonance."

Theorem 3. Suppose $f(x)$ is an odd function of $x$ and $|f(x)|=o(|x|)$, then Equation (3) has $n$ distinct one parameter families of periodic solutions $x_{i}(R)(i=1, \cdots, n)$ such that the period of $x_{i}(R), T_{i}(R) \rightarrow\left(2 \pi / \beta_{i}\right)$ as $R \rightarrow 0$. These families exist for large $R$ provided $f(x) x>0$ for $x \neq 0$.

REMARKS ON PROOF. The change of variables $t=\lambda s$ introduces the parameter $\lambda$ into the Equation (3). We consider "so-called" generalized periodic solutions $x(s)$ lying in the Hilbert space of odd, $2 \pi$ periodic, absolutely continuous functions which possess a square integrable derivative (see [2, Part 2, Chapter 3]).

3. Application to nonlinear Dirichlet problems. Let $\Omega$ be a bounded domain in $R^{N}$ with boundary $\partial \Omega$. Then the results of Theorems 1 and 2 apply to systems of the divergence form

$$
\begin{gathered}
\sum_{|\alpha| \leq m} D^{\alpha}\left(A_{\alpha}\left(x, u, \cdots, D^{m} u\right)\right)=\lambda \sum_{|\alpha| \leq m-1} D^{\alpha} B_{\alpha}\left(x, u, \cdots, D^{m-1} u\right), \\
\left.D^{\alpha} u\right|_{\partial_{\Omega}}=0, \quad|\alpha| \leqq m-1,
\end{gathered}
$$

provided the functions $A_{\alpha}$ and $B_{\alpha}$

(a) satisfy appropriate growth, ellipticity and continuity assumptions,

(b) arise as Euler-Lagrange equations for a functional of the form $\int_{\Omega}\left\{A\left(x, u, \cdots, D^{m} u\right)-\lambda B\left(x, u, \cdots, D^{m-1} u\right)\right\}$, and

(c) are odd functions of $u, D u, \cdots, D^{m} u$. For example, consider the simple quasi-linear system (for $\partial \Omega$ piecewise smooth). ( $N=2$ or 3 .)

$$
\begin{aligned}
\Delta u+\lambda\left\{u \pm u^{3}\right\} & =0 \text { in } \Omega \\
\left.u\right|_{\partial \Omega} & =0 .
\end{aligned}
$$

Suppose the eigenvalues of the Laplace operator $\Delta$ over $\Omega$ subject to null boundary conditions are denoted $\lambda_{1} \leqq \lambda_{2} \leqq \lambda_{3} \leqq \cdots \leqq \lambda_{n} \leqq \cdots$. Then the following result holds.

Theorem 4. Suppose $\lambda_{n}$ (as described above) is an eigenvalue of multiplicity $p$, then (5) \pm has at least $p$ one parameter families of solutions $\left(u_{n, i}(R), \lambda_{n, i}(R)\right)(i=1, \cdots, p)$ such that

(a) $\left(u_{n, i}(R), \lambda_{n, i}(R)\right) \rightarrow\left(0, \lambda_{n}\right)$ as $R \rightarrow 0$;

(b) $\lambda_{n, i}(R)$ depend continuously on $R$ for $R$ sufficiently small;

(c) $(1 / 2) \int_{\Omega}\left|\operatorname{grad} u_{n, i}(R)\right|^{2}=R$;

(d) $\left(u_{n, i}(R), \lambda_{n, i}(R)\right)$ can be continued as functions of $R$ to the open interval $(0, \infty)$, in $(5) \pm$. 
REMARK. The functions $\left\{u_{1,1}(R), 0<R<\infty\right\}$ for (5)-

(i) are the unique positive solutions of (5) for fixed $\lambda \in\left(\lambda_{1}, \infty\right)$;

(ii) tend uniformly to 1 as $R \rightarrow \infty$ apart from a small boundarylayer set concentrated near $\partial \Omega$.

\section{Bibliography}

1. M. S. Berger, On nonlinear perturbations of the eigenvalues of a compact selfadjoint operator, Bull. Amer. Math. Soc. 73 (1967), 704-708.

2. L. Bers, F. John and M. Schechter, Partial differential equations, Wiley, New York, 1964.

3. E. Hille and R. Phillips, Functional analysis and semigroups, Amer. Math. Soc. Colloq. Publ., vol. 31, Amer. Math. Soc., Providence, R. I., 1957.

University of Minnesota, Minneapolis, Minnesota 55455 